\title{
INFLUENCIA DEL CONTENIDO DE HUMEDAD DE EQUILIBRIO EN SORCIÓN Y DESORCIÓN SOBRE LA DUREZA DE CUATRO ESPECIES MADERABLES PLANTADAS EN COLOMBIA
}

Palabras clave: Contenido de humedad de equilibrio, dureza, sorción, desorción, Eucalyptus pellita, Eucalyptus tereticornis, Pinus caribaea, Tectona grandis.

Key words: Equilibrium moisture content, hardness, sortion, desorption, , Eucalyptus pellita, Eucalyptus tereticornis, Pinus caribaea, Tectona grandis.

\author{
Andrea Tatiana Pino Rodríguez ${ }^{1}$ \\ Yesenia Vásquez, Aguilera ${ }^{2}$ \\ César Polanco Tapia
}

\section{RESUMEN}

El contenido de humedad y la variación del mismo es uno de los factores que más influencia tiene sobre las propiedades mecánicas de la madera; por lo cual es necesario conocer la incidencia de estas fluctuaciones. La dureza, definida como la resistencia que presenta una madera a la penetración, las abolladuras y al desgaste, es fuertemente dependiente del contenido de humedad obtenido en el proceso de secado; así ésta puede verse afectada por los fenómenos de sorción y desorción. Se evaluó el efecto del contenido de humedad de equilibrio en sorción y desorción sobre la dureza para las especies Eucalyptus pellita, Eucalyptus tereticornis, Pinus caribaea y Tectona grandis, tomando como indicador la prueba de dureza, según el Comité Panamericano de Normas Técnicas 465. Se ajustaron los contenidos de humedad al $12 \%$ y por medio de correlaciones se estableció la influencia de la constitución anatómica sobre dicha propiedad. El análisis de la información se hizo a través de ANAVA de doble vía.
Los resultados obtenidos mostraron una dureza superior en desorción respecto a sorción; debido en parte a las condiciones que afectaron las maderas en el proceso de secado las cuales incidieron en un debilitamiento de las estructuras anatómicas y por consiguiente la desnaturalización de la madera.

\begin{abstract}
The moisture content and the variation of he himself are one of the factors that more influence has on the mechanical properties of the wood; thus it is necessary to know the incidence these fluctuations. The hardness, defined as the resistance that presents a wood to the penetration, the dents and to the wearing down; is strongly employee of the moisture content obtained in the drying process; which can be seen affected by the phenomena of sortion and desorption. Whit the present investigation one evaluated the effect of the equilibrium moisture content was evaluated on the hardness for the species Eucalyptus pellita, Eucalyptus tereticornis,
\end{abstract}

1 Ingeniera Forestal. Universidad Distrital "Francisco José de Caldas". Facultad de Medio Ambiente y Recursos Naturales. tatianaespora@ yahoo.es

2 Ingeniera Forestal. Universidad Distrital "Francisco José de Caldas”. Facultad de Medio Ambiente y Recursos Naturales. yeseniavasquezaguilera@yahoo.com.ar

3 Ingeniero Forestal. Universidad Distrital "Francisco José de Caldas". Director de la investigación. Director operaciones industriales Reforestadota de la costa S.A. cesarp@refocosta.com 
Pinus caribaea and Tectona grandis; taking like indicator the test of hardness, according to COPANT 465. The moisture contents adjusted to $12 \%$ and by means of correlations the influence of the anatomical constitution settled down on this property. The analysis of the information became through ANAVA of double via. The obtained results showed a superior hardness in desorption with respect to sortion; had to the conditions that affected the wood in the drying partly process which affected a weakening of the anatomical structures and therefore the denaturation of the wood.

\section{INTRODUCCIÓN}

El contenido de humedad (C.H.\%) es el porcentaje en peso del agua presente en la madera con respecto al peso de la madera seca (JUNAC 1988). Los cambios de C.H.\% afectan notoriamente la respuesta de la madera según sean las cargas a las que se encuentran sometidas. Lógicamente, esta influencia sobre las propiedades es diferente para cada especie y no sólo depende del contenido de humedad, sino también de la densidad, de la estructura anatómica de las maderas, de la posición de la troza en el árbol o de la cercanía de la misma a la médula (Arévalo \& Hernández 2004).

La dureza de la madera, definida como la resistencia que presenta a la penetración, a las abolladuras y al desgaste (COPANT 1972), depende fuertemente del contenido de humedad obtenido en el proceso de secado. De tal forma, la dureza puede verse afectada por los fenómenos de sorción y desorción, en donde el primero ocurre cuando una madera completamente seca, en contacto con vapor de agua, lo absorbe hasta que se produce un estado de equilibrio con el ambiente, mientras que el segundo se presenta cuando la madera, completamente saturada, en un ambiente seco, pierde agua hasta que llega a un estado de equilibrio con el ambiente. (Kollmann 1959).
Diferentes investigaciones han determinado las propiedades físico mecánicas de las especies Eucalyptus pellita F. Muelle. (Ruiz \& Rodríguez 2006), Pinus caribaea Morelet (Grisales \& Argüello 2005), Tectona grandis L. f. y Eucalyptus tereticornis Sm. (Cifuentes $\&$ Medina 2005), los cuales nos proporcionaron los valores de dureza necesarios para poder comparar la variación con las condiciones de humedad.

Valero et al. (2002) establecieron la relación entre la anatomía y las propiedades físico mecánicas de la madera de la especie Tectona grandis (2002) observando que existía una relación entre las características anatómicas (proporción de poros, radios, parénquima y fibras) con las propiedades físicas (densidad, peso específico, contracciones y T/R) y mecánicas (flexión, compresión paralela, compresión perpendicular, dureza, cizallamiento y tenacidad) de la madera. Encontraron además, con un nivel de significancia del 95\%, que existían diferencias relevantes entre las tres condiciones de la madera utilizadas en las pruebas (verde, seca al aire, seca al horno) sobre la compresión perpendicular, dureza y tenacidad.

Algunos investigadores en Venezuela han hecho referencia a la importancia de conocer la anatomía de la madera para poder realizar inferencias sobre las propiedades físicas y mecánicas, técnicas de procesamiento y utilización de la madera, debido a que el comportamiento de la madera es un reflejo de sus características anatómicas (Espinoza de Pernía y León 2001).

Dado que el objetivo de esta investigación es determinar la influencia del contenido de humedad de equilibrio en sorción y desorción, sobre la dureza de cuatro especies maderables plantadas en Colombia, se tuvieron en cuenta variables como la procedencia para la especie E. pellita y la edad para las especies $T$. grandis y $P$. caribaea. La variable de procedencia 
corresponde a la semilla de E. pellita, una proveniente de Papua Nueva Guinea y la otra de origen nacional. En cuanto a la variable de la edad se presentan dos edades de plantaciones, que casualmente son del mismo año para ambas especies, 1982 y 1987 . De la especie $E$. tereticornis solo se tenían muestras de una procedencia y edad, por lo tanto no hay variables adicionales para esta especie. Es decir, aunque son cuatro especies, al tener en cuenta estos aspectos consideraremos como variables a las siete combinaciones resultantes. Adicionalmente, se evaluó la incidencia de la constitución anatómica sobre la resistencia en dureza que alcanza la madera.

\section{MATERIALES}

\section{ESPECIES ESTUDIADAS}

Eucalyptus pellita. Árbol perteneciente a la familia Myrtaceae, que llega hasta $40 \mathrm{~m}$ de altura, y $1 \mathrm{~m}$ de diámetro. Es originario de Australia y Nueva Guinea, donde se encuentra en bosques abiertos. Crece desde los cero hasta los $600 \mathrm{msnm}$, con temperaturas que oscilan entre los $9^{\circ} \mathrm{C}$ a $\operatorname{los} 30^{\circ} \mathrm{C}$ y precipitaciones que varían entre los 900 a 2200 mm (IPEF 2004). Esta especie se emplea para construcciones, mobiliarios, chapas, aglomerados, energía, y laminados (Betancourt 1987). Las propiedades físico-mecánicas de esta especie se muestran en la Tabla 1.

Eucalyptus tereticornis. Esta especie pertenece a la familia Myrtaceae y es originaria de Australia. Se encuentra distribuida desde Australia hasta la costa de Nueva Guinea. Crece en climas templados a tropicales entre los $6^{\circ}$ $\mathrm{C}$ y $38^{\circ} \mathrm{C}$ en alturas desde los cero a los 1000 msnm Árbol de gran porte que alcanza hasta $45 \mathrm{~m}$ de altura. Se usa principalmente la madera rolliza en postes, producción de pulpa y leña (Conif 1996); así como en soleras, pisos, encofrados, cerchas, andamios, parales y cons- trucciones livianas. La madera es difícil de secar al aire libre, presentando deformaciones y agrietamientos en el proceso de secado. Es moderadamente durable, y resistente al ataque de hongos (Refocosta 2005). Las propiedades físico-mecánicas se muestran en la Tabla 1.

Pinus caribaea. Árbol que pertenece a la familia Pinaceae. Es un pino tropical que crece de manera natural a bajas elevaciones. Es un árbol majestuoso y alto que crece rápidamente y produce una madera resinosa útil para la construcción y productos de papel (Francis 1992). Crece desde nivel del mar hasta los $1500 \mathrm{msnm}$, con temperaturas entre $\operatorname{los} 22^{\circ}$ y $26^{\circ} \mathrm{C}$. Cuando la madera se trabaja húmeda presenta dificultades por el alto contenido de resinas naturales, resiste las termitas y seca relativamente fácil al aire libre, presentando leves deformaciones. La madera se usa en construcción, para pulpa, aserrío, fabricación de muebles y resinas (Conif 1995). Algunas de sus propiedades se ilustran en la Tabla 1.

Tectona grandis. Árbol de la familia Verbenaceae, originario de la India. Puede alcanzar alturas de hasta $40 \mathrm{~m}$, con un tronco de 1 a $1.5 \mathrm{~m}$ de diámetro. Aunque se adapta a una gran variedad de suelos requiere de climas con una estación seca bien definida, temperaturas medias anuales entre 22 y $28^{\circ} \mathrm{C}$, una precipitación media anual de 1250 a $1500 \mathrm{~mm}$ y altitudes entre los 0 a $1000 \mathrm{~m}$ (Weaver 1993). La madera es sólida, de fibra compacta y de secado lento; es considerada madera fina y dura, cualidad muy apreciada para diversos usos. Además, se caracteriza por contener sílice, es fácil de trabajar, secar y preservar, con una buena durabilidad natural y estabilidad dimensional (Weaver 1993). El secado al horno es bueno, sin tendencia a rajaduras o alabeos. No es corrosiva, tiene resistencia a las termitas, los hongos y a la intemperie (Original Teka Art 2005). Algunas propiedades de la especie, se muestran en la Tabla 1. 
Tabla 1. Propiedades físico mecánicas de las especies estudiadas.

\begin{tabular}{|c|c|c|c|c|c|c|c|}
\hline Especie & $\begin{array}{l}\text { Densidad } \\
\text { básica } \\
\left(\mathrm{gr} / \mathrm{cm}^{3}\right)\end{array}$ & $\begin{array}{c}\text { Grupo } \\
\text { (NTC 2500 } \\
\text { Icontec) }\end{array}$ & $\begin{array}{l}\text { Módulo } \\
\text { elasticidad } \\
\text { mínimo } \\
\left(\mathbf{k g} / \mathrm{cm}^{2}\right)\end{array}$ & $\begin{array}{l}\text { Esfuerzo a } \\
\text { flexión } \\
\text { radial } \\
\left(\mathrm{kg} / \mathrm{cm}^{2}\right)\end{array}$ & $\begin{array}{c}\text { Esfuerzo a } \\
\text { comprensión } \\
\text { paralela } \\
\left(\mathrm{kg} / \mathrm{cm}^{2}\right)\end{array}$ & $\begin{array}{c}\text { Esfuerzo a } \\
\text { comprensión } \\
\text { perpendicular } \\
\left(\mathrm{kg} / \mathrm{cm}^{2}\right)\end{array}$ & Fuente \\
\hline E. pellita & 0.96 & B & N.D & 305.91 & 689.32 & 216.51 & Ruiz \& Rodríguez, 2006 \\
\hline E. tereticornis & 0.85 & B & 125,65 & 540.27 & 459.99 & 75.54 & Cifuentes \& Medina, 2005 \\
\hline P. caribaea & 0.48 & C & N.D & 100 & 80 & 25 & Grisales \& Argüello, 2005 \\
\hline T. grandis & 0.61 & B & 100.51 & 121.66 & 194.71 & 25.95 & Cifuentes \& Medina, 2005 \\
\hline
\end{tabular}

\section{PROCEDENCIA Y PREPARACIÓN DE LA MADERA}

La madera de todas las especies provino de plantaciones de la Reforestadora de la Costa (REFOCOSTA). Los lotes de madera correspondientes a las especies: $T$. grandis y $E$. tereticornis se obtuvieron del área del proyecto denominado "La Gloria", Municipio Sabanas de San Ángel, Departamento de Magdalena, Colombia. Las especies P. caribaea y E. pellita provinieron del Municipio de Villanueva, Departamento de Casanare.El almacenamiento y procesamiento de las trozas se realizó en el laboratorio de Tecnología de maderas José Anatolio Lastra Rivera perteneciente a la Universidad Distrital Francisco José de Caldas, en Bogotá.

\section{METODOLOGÍA}

Se realizaron cortes a las trozas de madera, para obtener probetas de $5 \times 4 \times 12 \mathrm{~cm}$ teniendo en cuenta tres planos anatómicos (radial, transversal y tangencial). Se utilizaron en promedio 46 probetas por cada especie (lote) orientadas respecto a los anillos de crecimiento (Kollmann 1975).

Para la descripción macroscópica de las especies se seleccionaron seis probetas al azar de cada especie, y se caracterizaron los siguientes parámetros: color, transición entre albura y duramen, olor, sabor, dirección del grano, textura, veteado, distribución de la porosidad, visibilidad de los anillos de crecimiento, poros, radios, parénquima longitudinal y radios, ausencia o presencia de Ripple Marks ó estratificación de radios. La evaluación de estos parámetros fue realizada con la simple observación y a veces con ayuda de una lupa de aumento de 10x. Los colores fueron clasificados de acuerdo a la tabla de colores Munsell y los rangos de clasificación son los establecidos por el Laboratorio de Tecnología de Maderas "José Anatolio Lastra Rivera" en su formato académico No. F-06 de 2004.

\section{CARACTERIZACIÓN MICROSCÓPICA DE LAS ESPECIES}

Se seleccionaron al azar tres probetas de cada especie con su respectiva variable, es decir para E. pellita ambas procedencias y las dos edades para $T$. grandis y $P$. caribaea. Se realizaron cortes de $2 \times 2 \times 2 \mathrm{~cm}$ aproximadamente, que luego se dejaron sumergidos en agua corriente por alrededor de seis semanas. Después del ablandamiento se realizaron cortes con el empleo del micrótomo, para obtener muestras con espesores entre 10 y $20 \mu \mathrm{m}$. El tratamiento siguiente consistió en usar diferentes reactivos que prepararon el material para ser montado y secado, y finalmente poderlo observar en el microscopio. Los reactivos y los tiempos de permanencia fueron: fucsina básica (5 minutos), ácido pícrico (24 horas), etanol en concentración $25 \%, 50 \%, 75 \%$ y 
96\% (10 minutos en cada uno), Xilol- Etanol (10 minutos) y Xilol (10 minutos). Luego de esto se realizó el montaje en la lámina portaobjetos usando como pegamento el bálsamo de Canadá. Posteriormente las laminillas permanecieron en la estufa por un lapso de 24 horas a una temperatura de aproximadamente $103^{\circ}$ C. Luego se realizó el reconocimiento de los planos que componen las laminillas, determinando las principales estructuras de sostén y aquellas que ocasionan el debilitamiento. Se identificaron los siguientes rasgos anatómicos: tipo de fibras y su longitud, vasos, traqueídas, tipo de punteaduras, patrones en la variación del tamaño de los poros y su disposición, forma, contenido, cantidad y tamaño. Además se definió el tipo de parénquima (apotraqueal o paratraqueal), longitud de los segmentos vasculares, evaluación de los radios según el número de células de ancho, cantidad, tipos de células, estratificación, contenidos y cantidad.

\section{PRUEBAS DE DUREZA}

La madera de las trozas se secó al aire libre, hasta adquirir un Contenido de Humedad del $12 \%$ aproximadamente. Este tratamiento se denominó desorción; momento en el cual se realizó la primera prueba de dureza (Argüello et al 2005, Cifuentes et al 2005 \& Ruiz et al 2006). Para la prueba de dureza en sorción se utilizaron probetas del mismo muestreo, las cuales se sometieron a un tratamiento previo que consistió en el secado total de la madera en estufa hasta alcanzar un C.H.\% equivalente al estado anhidro y nuevamente esperando que llegaran al equilibrio en el mismo ambiente de una manera natural (Pino \& Vásquez 2007). Las pruebas se ejecutaron en la máquina Universal que penetra en las probetas $1 \mathrm{~cm}^{2}$, a una velocidad constante de $6 \mathrm{~mm} / \mathrm{min}$, con una carga aplicada de $3000 \mathrm{~kg}$ sobre cada plano (COPANT 465 1972).

\section{DETERMINACIÓN DEL CONTENIDO DE HUMEDAD}

Se realizaron cortes a todas las probetas sacando muestras de $5 \times 4 \times 2 \mathrm{~cm}$. Estas secciones fueron secadas nuevamente y pesadas en promedio 4 veces hasta encontrar un peso constante para cada variable. Estos datos se remplazaron en la siguiente fórmula (JUNAC 1988):

$$
\text { C.H. } \%=\frac{\mathrm{Ph}-\mathrm{Pa}}{\mathrm{Pa}} \times 100 \text { donde, }
$$

Ph: Peso húmedo (peso inicial)

Pa: Peso anhidro (peso final)

\section{AJUSTE DE DUREZA AL $12 \%$ DE CONTENIDO DE HUMEDAD}

Los resultados obtenidos en las pruebas de dureza fueron ajustados a un contenido de humedad del 12\% según los factores de corrección descritos por Silvester (1967). Para los datos de dureza correspondientes a las partes extremas de las probetas ó a los planos transversales se empleó un factor del $4 \%$ y en planos laterales (radial y tangencial) un factor de $2.5 \%$, por cada $1 \%$ de variación del contenido de humedad, asumiendo una relación lineal tanto en Sorción como en Desorción.

\section{ANÁLISIS DE DATOS}

Se implementó un Diseño en Bloques Completos al Azar con diferente número de repeticiones (Steel \& Torrie 1985). En un primer tipo de análisis, los bloques correspondieron a las especies estudiadas relacionadas por plano anatómico y el contenido de humedad en sorción y desorción. En un segundo tipo de análisis, los bloques correspondieron a las especies relacionadas con la procedencia de la semilla, la edad de plantación y el contenido de humedad en sorción y desorción. 


\section{RESULTADOS Y DISCUSIÓN}

\section{DUREZA EN SORCIÓN \\ Y DESORCIÓN POR ESPECIE}

En términos generales los resultados muestran que existen diferencias significativas entre la dureza en sorción y desorción para todas las especies (RV 18,17 > 8.10) siendo mayor la dureza en desorción con respecto a la dureza en sorción (Figura 1). E. pellita presentó mayores valores en desorción con respecto a la sorción de manera significativa debido al colapso ó hundimiento de las fibras generado en la primera etapa de la prueba de sorción (secado al horno) a causa de la marcada propensión a rajaduras que posee esta especie. De igual forma, E. tereticornis mostró un considerable descenso en la dureza de los extremos en las pruebas de sorción (Figura 1). Esto nos lleva a suponer que existe una susceptibilidad implí- cita de estas especies al colapso, en donde una pérdida acelerada del agua libre y una baja resistencia de la pared celular conllevarán a una menor resistencia.

Respecto a $T$. grandis, se obtuvieron valores superiores de dureza en la plantación de 1982 que en la de 1987 (Figura 1). Esta diferencia probablemente es debida a la procedencia y a la edad. Cuando la madera es proveniente de árboles jóvenes, la durabilidad es menor que la madera en etapa madura, pues se hace progresivo un aumento en las propiedades mecánicas a medida que el árbol envejece, por una mayor lignificación, haciéndose más evidentes las diferencias en las áreas próximas a la médula (Castro et al. 2001 \& Valero et al. 2002).

En $P$. caribaea, la tendencia para los años 1982 y 1987 estuvo sujeta a la baja densidad del material, la homogeneidad de las estruc-

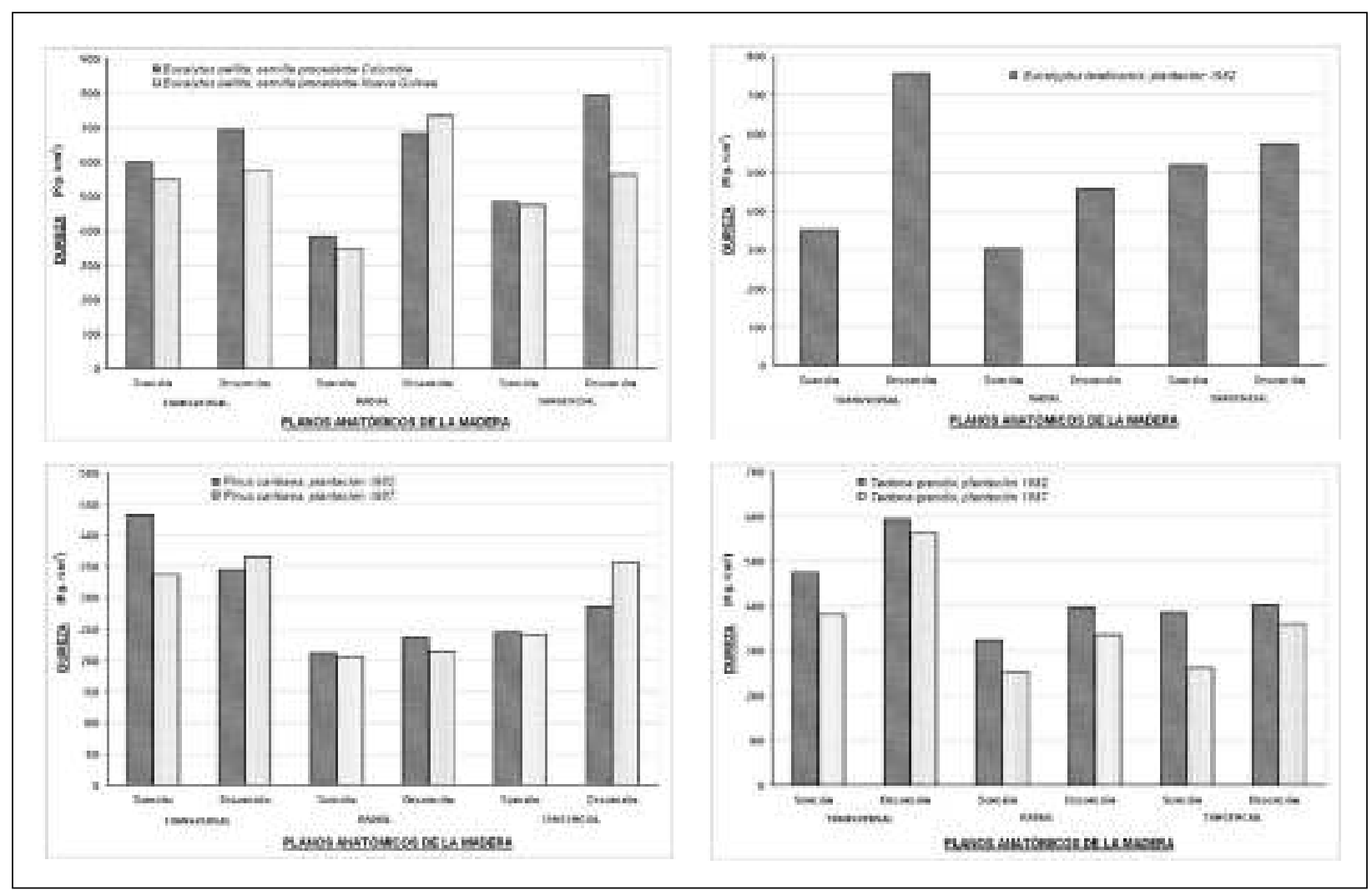

Figura 1. Registros de dureza en sorción y desorción de T. grandis, P. caribaea, E. pelita y E. tereticornis a nivel de planos tangencial, radial, y transversal normalizados a un Contenido de Humedad del $12 \%$. 
turas anatómicas y su distribución, por lo que no se encontró un patrón claro (Figura 1). La densidad de esta especie varía por ende con la proporción de anillos de crecimiento de madera tardía y temprana, y el porcentaje de resina depositado en ella.

\section{INFLUENCIA GENERAL DE LOS ELEMENTOS ANATÓMICOS}

Las estructuras que determinan la resistencia de las especies se evidencian en primer lugar en los elementos que sirven de sostén como las fibras (latifoliadas) o traqueídas (coniferas) y, en segundo lugar, en los elementos que generan debilidad como los vasos, su longitud, el parénquima longitudinal y radial y, en algunos casos muy específicos, radios de tamaño variable y altura considerable (Figura 4).

Al comparar los resultados entre los planos anatómicos de las especies, se registran mayores valores de dureza en los planos transversales y menores valores en los planos tangenciales y radiales (Figura 1). Por otro lado, se presentan diferencias significativas entre los planos transversales (RV $11.51>8.10$, $\mathrm{p}<0.01$ ), lo cual es particularmente evidente en las especies que tienen una diferenciación definida entre las células de madera temprana y tardía, en las que se presenta un gradiente de resistencia sobre todo en el plano transversal. Tal es el caso de $P$. caribaea y coincide con los hallazgos de otros autores, quienes han sugerido que para las coníferas "las traqueídas que se desarrollan en la madera tardía poseen paredes más gruesas y diámetro radial más reducido que el las traqueídas de madera temprana; lo cual refleja diferencias de densidad en el mismo anillo de crecimiento, derivando una menor densidad en la madera temprana y una mayor densidad en la madera tardía" (Espinoza de Pernia \& León 2001). Esto se observa claramente en los anillos de crecimiento de madera temprana y tardía de $P$. caribaea plantado en 1987; en cada una de las etapas de crecimiento se tienen características definidas $\mathrm{y}$ diferenciales en los elementos anatómicos (grosor de las paredes, cantidad) lo que hace que se generen variaciones lineales en cuanto a la dureza (Figura 2).

P. caribea presenta una baja susceptibilidad al colapso, pues el excesivo número y tamaño de las punteaduras asociadas a coníferas amortiguan el efecto de una salida rápida del agua libre dada la oclusión de la membrana de cierre sobre la abertura. Aspecto que permite mantener la integridad de los tejidos conformados por traqueídas.

La especie $T$. grandis se comporta de manera similar a $P$. caribaea al presentar un gradiente sobre cada uno de los planos anatómicos, debido a la presencia, cantidad y distribución de los elementos anatómicos que favorecen o contrarrestan la acción de resistencia de la pieza. Para la confiera (P. caribaea) su marcada diferenciación entre la madera temprana y la madera tardía y para la latifoliada (T. grandis), la diferenciación de los tamaños de los poros, que aunque no es muy marcada (porosidad semicircular) genera variaciones en los resultados de dureza como se ve en la Figura 3.

\section{INFLUENCIA DE LOS ELEMENTOS ANATÓMICOS POR PLANO}

Se encontró que la dureza en desorción fue significativamente mayor para todas las especies en el plano radial (RV 24.51 > 8.10, Figura 1). Esto probablemente se debe a la oposición que proporcionan las fibras al unirse con los radios, se registran los mayores valores de dureza como ha sido sugerido en anteriores estudios. No obstante, una distribución uniforme de los radios origina una superior estabilidad dimensional, lo cual no indica directamente una mayor resistencia en dureza. 

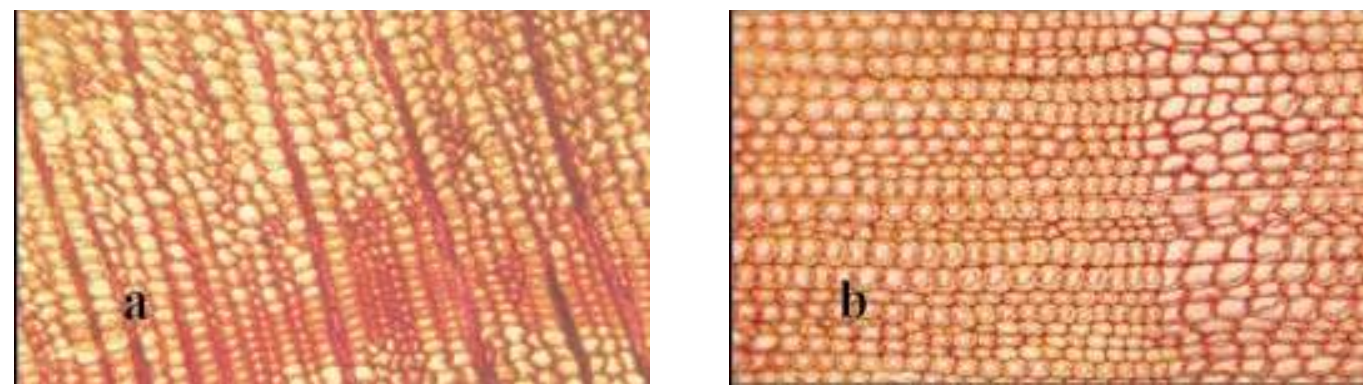

Figura 2. Corte del plano transversal de $P$. caribaea plantado en 1987 a) detalle de los anillos de crecimiento de madera temprana y b) detalle de los anillos de crecimiento de madera tardía.

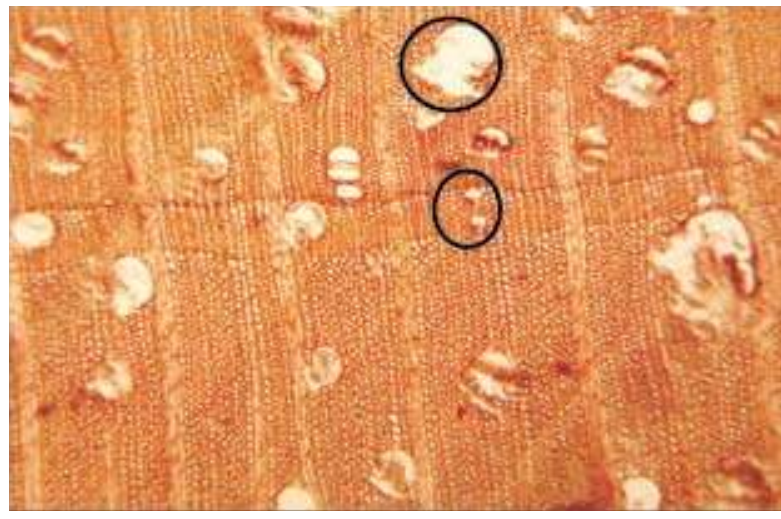

Figura 3. Evidencia de los tamaños diferenciales de los poros en los anillos de crecimiento de Tectona grandis plantada en 1982 .

De forma similar que para el plano radial, en el plano tangencial la dureza en desorción también fue significativamente mayor (RV 26.86> 8.10, Figura 1). En este caso, la dureza puede fluctuar de acuerdo principalmente a la altura de los radios, su grosor y la cantidad. De tal forma que entre más numerosos, más “amarre" se dará; así como radios más homogéneos, más pequeños y distribuidos uniformemente proveen un mayor coeficiente de estabilidad dimensional, es decir un comportamiento más estable de las estructuras.

En el plano trasversal, la dureza, está relacionada con la cantidad de parénquima, la ubicación y el tamaño de los poros y el grosor de las paredes de las fibras, así como la longitud de las mismas. A medida que el parénquima sea más abundante, su resistencia será menor entre mayor sea el tamaño de los poros y mas diferenciados sean entre si, y se presentaran menores resultados en la dureza. Entre más gruesas sean las paredes de las fibras, será mayor el esfuerzo de dureza.

\section{EFECTO DE LA PROCEDENCIA DE LA SEMILLA EN LA DUREZA}

Para E. pellita y T. grandis se encontró que la procedencia de la semilla genera diferencias entre la dureza en sorción y desorción (RV $10.90>6.61 \mathrm{p}<0.05$, RV 18,40 > 16.3, p < 0.01 , respectivamente). Por el contrario, en $P$. caribaea no se encontraron diferencias significativas entre procedencias (RV 18,17 > 8.10, $\mathrm{p}>0.05)$. Las condiciones climáticas del lugar donde se encuentra el árbol padre, es decir donde se produce la semilla, influyen directamente sobre el germoplasma producido. Para determinar las causas de la diferencia entre las procedencias de las especies es necesario profundizar en la influencia de las condiciones locales del área de crecimiento, sobre las propiedades de la madera; considerando los efectos que pueda tener en su crecimiento.

\section{CONCLUSIONES}

De acuerdo a los resultados obtenidos en esta investigación el comportamiento generalizado de la dureza fue superior en desorción que en sorción, por lo cual se considera más efectivo trabajar con las especies objeto de 
estudio en desorción, cuando van a ser sometidas a la prueba de dureza. Es decir que las maderas que fueron afectadas por un proceso de pérdida de humedad para llegar al equilibrio presentan una mayor dureza o resistencia a la penetración de un cuerpo externo que aquellas que ganaron humedad para llegar al equilibrio.

El cambio acelerado de las condiciones a las que fueron sometidas las maderas en el proceso de secado, ocasionó un debilitamiento de las estructuras anatómicas y por consiguiente su desnaturalización, lo cual incidió directamente en la resistencia y obviamente en el resultado de las pruebas, lo cual se hizo evidente en la ruptura de algunas de las estructuras a nivel microscópico y submicroscópico.

Las especies pertenecientes al género $E u$ calyptus, a causa de sus características propias, presentan mayor susceptibilidad al colapso, debido a la perdida acelerada del agua libre y a la baja resistencia de la pared celular.

La especie Tectona grandis, plantada en 1982 y en 1987 presentó un comportamiento similar respecto a la carga soportada. Sin embargo, la mayor resistencia se presentó en la especie de 25 años, ratificando que en la medida que se acerca a una edad madura, el árbol mejora sus propiedades mecánicas, dada una mayor lignificación y duraminización.

El Pinus caribaea plantado en 1982 y 1987 presentó los menores valores de resistencia en todos los tratamientos, esto debido a una menor densidad comparada con otras especies.

Los elementos anatómicos que más influyen en el momento de determinar la resistencia de la madera en dureza son el grosor de las paredes de las fibras en latífoliadas y traqueídas en coníferas, considerando a su vez que son predominantes en proporción en la constitución de la madera. Mientras las estructuras que determinan la susceptibilidad a la penetración están dadas por la abundante presencia: de parénquima longitudinal, poros, vasos y radios anchos.

\section{REFERENCIAS BIBLIOGRÁFICAS}

Arévalo, R. \& R. Hernández 2004. Efecto de la sorción de humedad en las propiedades físico - mecánicas de la madera de Caoba (Swietenia macrophylla King). Revista Colombia Forestal. 8: 17: 110.

Betancourt, B.A. 1987. Silvicultura especial de árboles maderables tropicales. Editorial Científico-Técnica, 427 pg. La Habana, Cuba.

Castro, F. \& J. Raigosa, 2001. Crecimiento y propiedades físicas de la madera de teca (Tectona grandis L.F.) de 17 años en San Joaquín de Abangares, Costa Rica. Comunicación técnica revista Forestal centro americana. 35: 19-24

Cifuentes, F. \& J. Medina, 2005. Determinación de las propiedades físicas, mecánicas y durabilidad natural de Teca (Tectona grandis) y Eucalipto (Eucalyptus tereticornis) procedencia Fundación (Magdalena). Trabajo Universidad distrital Francisco José de Caldas, Facultad de Medio ambiente y recursos Naturales, Laboratorio de Tecnología de maderas "José Anatolio Lastra Rivera”. 60 h. Bogotá, Colombia.

Comisión panamericana de normas técnicas (COPANT). 1972. Maderas. Método de determinación de la dureza. COPANT 465.

Corporación nacional de investigación y fomento forestal (CONIF) 1995. Coníferas: Guía técnica forestal. Editorial Ciudad Universitaria, $26 \mathrm{pg}$. 


\section{Corporación nacional de investigación y fo-} mento forestal (CONIF) 1996. latífoliadas zona baja: Guía técnica forestal. Editorial Ciudad Universitaria, $50 \mathrm{pg}$.

Espinoza de Pernía, N. \& W. J. León, 2001. Atlas para la identificación microscópica de maderas latífoliadas. Editorial consejo de Publicaciones. 78 pg. Mérida, Venezuela.

Francis, J. K. 1992. Pinus caribaea Morelet. Caribbean pine. Department of Agriculture, Forest Service, Southern Forest Experiment Station. 10 pg. New Orleans, United Estates.

Grisales, H. \& O. Arguello 2005. Determinación de las propiedades físicas, mecánicas del Pino (Pinus caribaea) procedencia Villanueva (Casanare). Trabajo Universidad distrital Francisco José de Caldas, Facultad de Medio ambiente y recursos Naturales, Laboratorio de Tecnología de maderas "José Anatolio Lastra Rivera". 70 h. Bogotá, Colombia.

Instituto de pesquisas e estudos florestais (IPEF). 2004. Chave de Identificação de Espécies Florestais. [en línea]. <http:// www.ipef.br/identificacao/cief/especies/ pellita.asp $>$

Junta del acuerdo de cartagena -JUNAC. 1988. Seminario para la Preservación de la Madera. Bogota. Junta del Acuerdo de Cartagena, grupo Andino.

Kollmann, F. 1959. Tecnología de la Madera y sus aplicaciones, Tomo I. 2. Editorial Ministerio de Agricultura, Instituto Forestal de investigaciones y experiencias y el servicio de la madera. 675 pg. Madrid, España.

Lastra, R. J. A. 1984. Mimeografía: Aspectos generales sobre la estructura anatómica, propiedades físico mecánicas y secado de la madera. Editorial Universidad Distrital Francisco José de Caldas. 60 pg Bogotá, Colombia.

Ruiz, H. \& J. D. Rodríguez 2006. Incidencia de dos procedencias sobre las propiedades físico mecánicas de Eucalyptus pellita F Muelle. caso Colombia y Papua Nueva Guinea. Trabajo de grado Universidad distrital Francisco José de Caldas, Facultad de Medio ambiente y recursos Naturales, Ingeniería Forestal. 74 h. Bogotá, Colombia

Silvester, F. T. 1967. It's Mechanical Properties and Factors Affecting its structural use. Pergamos Press LTD.

Steel, R. G. D. \& J. H. Torrie 1985. Bioestadística: principios y procedimientos. Editorial McGraw-Hill. 622 pg. Bogotá, Colombia.

Tomazello, M. 1985. Estructura anatómica de la madera de ocho especies de eucalipto plantadas en Brasil. Editorial Instituto de Pesquisas y Estudios Forestales (IPEF). Vol. 29 pg 25-36. Rio de Janeiro, Brasil.

Valero U. W., C. E. Reyesk, H. W. León \& J. D. Garay 2002. Relación entre anatomía y propiedades físico-mecánicas de la especie Tectona grandis proveniente de los llanos occidentales de Venezuela. Revista Forestal Universidad de Los Andes. [en línea]. http://www.saber.ula.ve/.../revistaforestal/ vol472/articulo3.pdf; 1:46:92

Weaver, P.L. 1993. Tectona grandis L.f. Teak. Department of Agriculture, Forest Service, Southern Forest Experiment Station. 18 pg. New Orleans, United Estates. 


\section{(a). Eucalyptus pellita semilla procedente de Colombia}

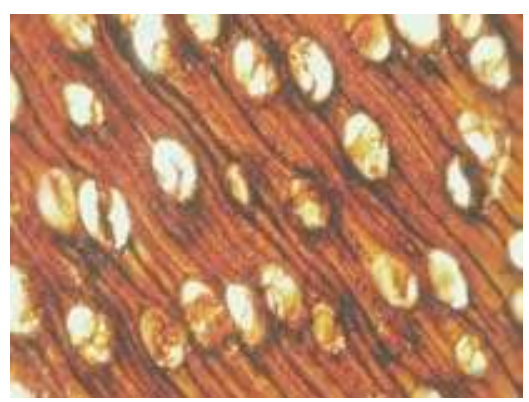

Plano Transversal $<9.1 \mathrm{x}>$

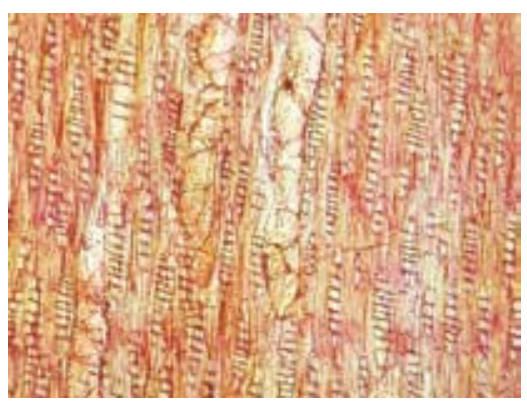

Plano Tangencial $<14 \mathrm{x}>$

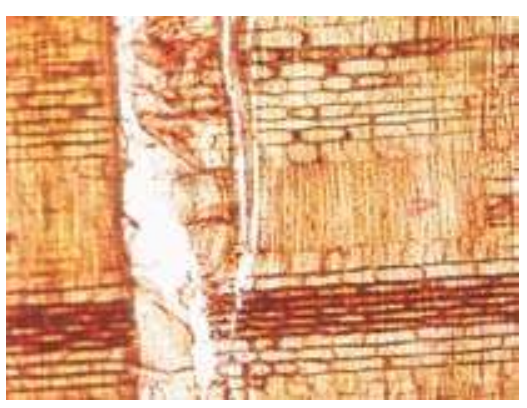

Plano Radial $<5.1 \mathrm{x}>$

\section{(b) Eucalyptus pellita semilla de procedencia Papua, Nueva Guinea}

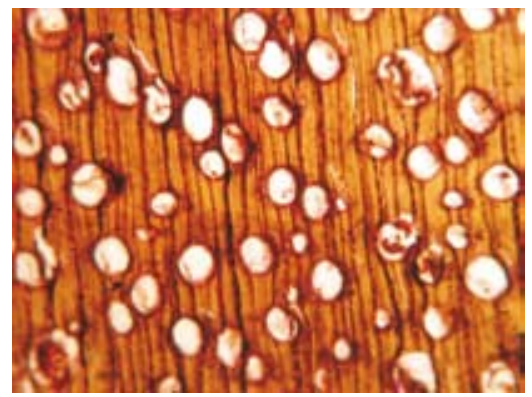

Plano Transversal $<9.1 \mathrm{x}>$

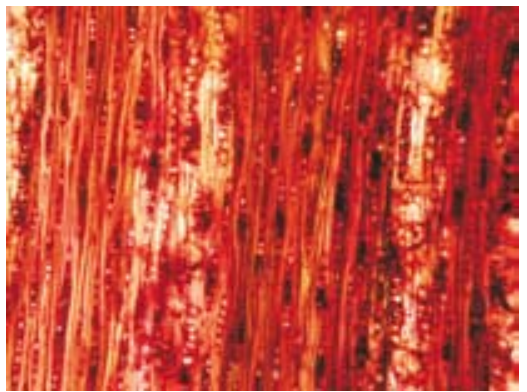

Plano Tangencial $<14 \mathrm{x}>$

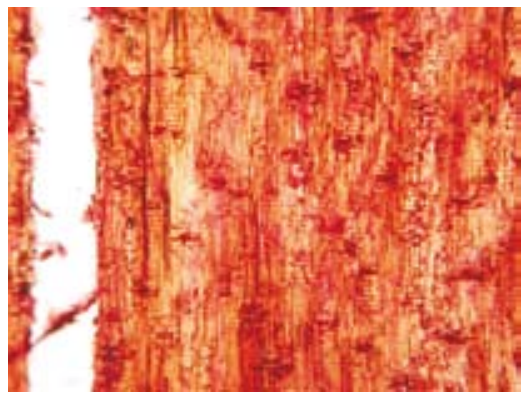

Plano Radial $<9.1 \mathrm{x}>$

\section{(c) Eucalyptus tereticornis plantado 1982}

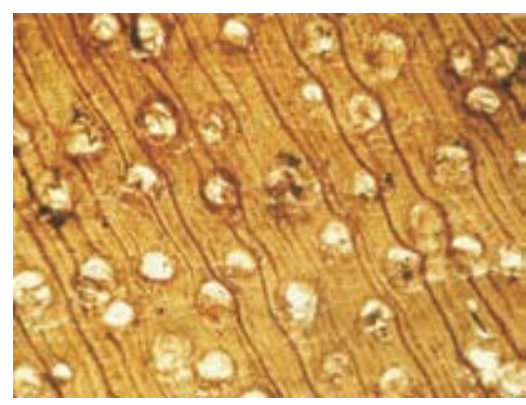

Plano Transversal $<9.1 \mathrm{x}>$

(d) Tectona grandis plantado 1982

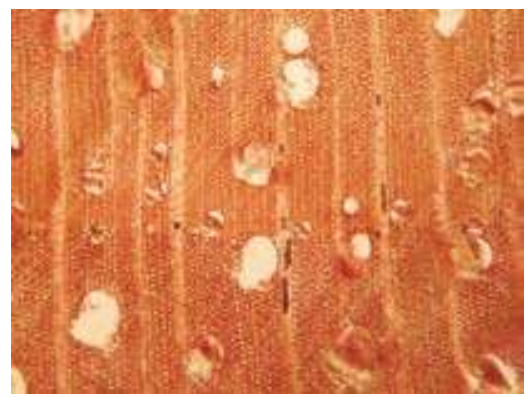

Plano Transversal $<10.1 \mathrm{x}>$

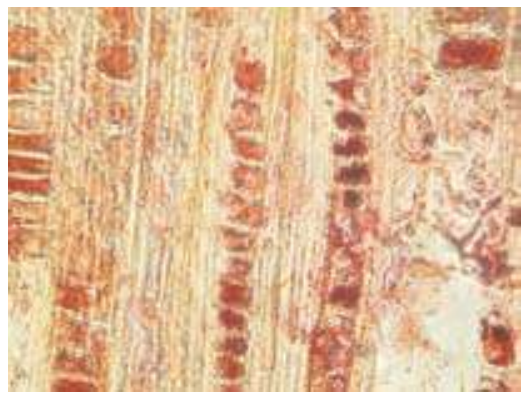

Plano Tangencial $<15.1 \mathrm{x}>$

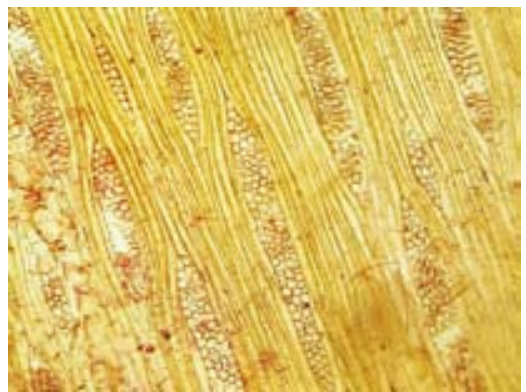

Plano Tangencial $<9 \mathrm{x}>$

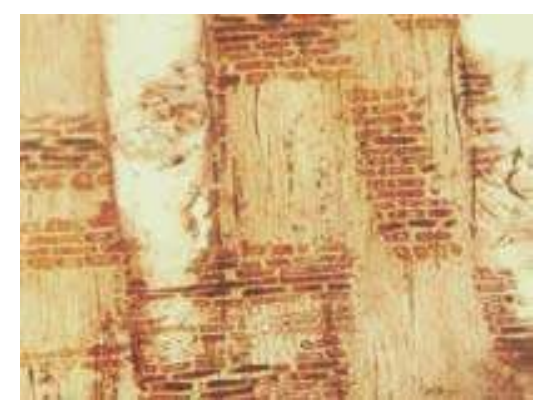

Plano Radial $<11.5 \mathrm{x}>$

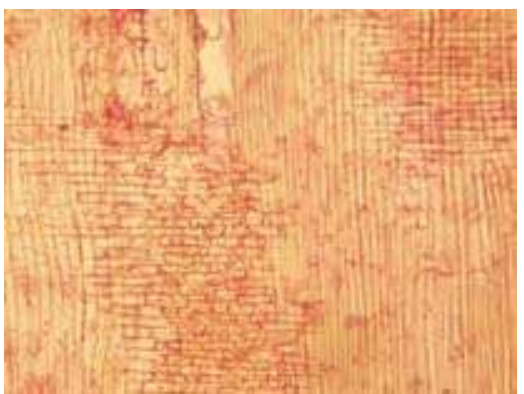

Plano Radial $<10.1 x>$

Figura 4. Fotografias microscopia de las especies estudiadas. 
(e) Tectona grandis plantada en 1987

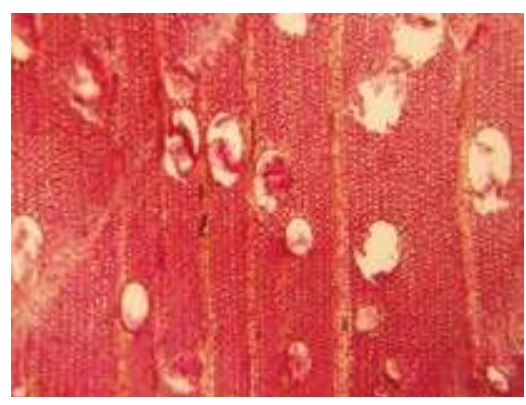

Plano Transversal $<10.1 \mathrm{x}>$

\section{(f) Pinus caribaea plantado en 1982}

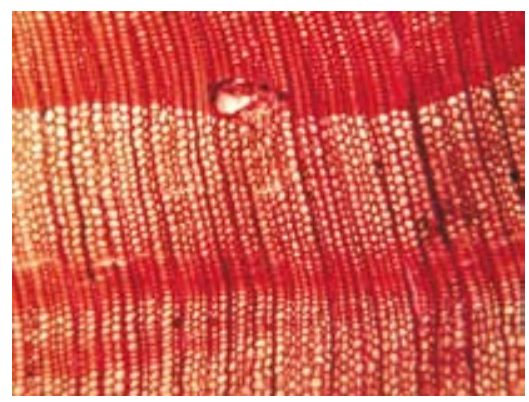

Plano Transversal $<10.1 \mathrm{x}>$

\section{(g) Pinus caribaea plantado en 1987}

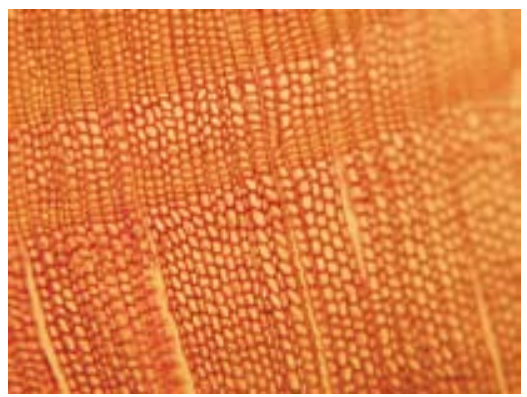

Plano Transversal $<10.1 \mathrm{x}>$

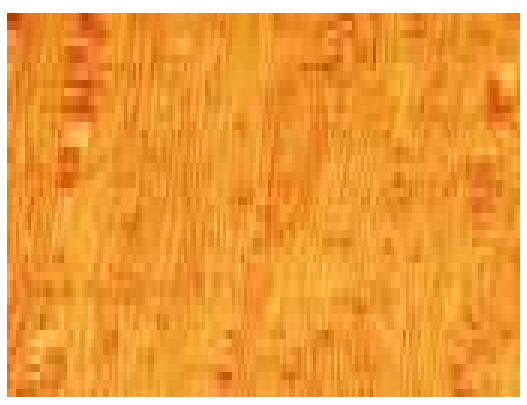

Plano Tangencial $<11.5 \mathrm{x}>$

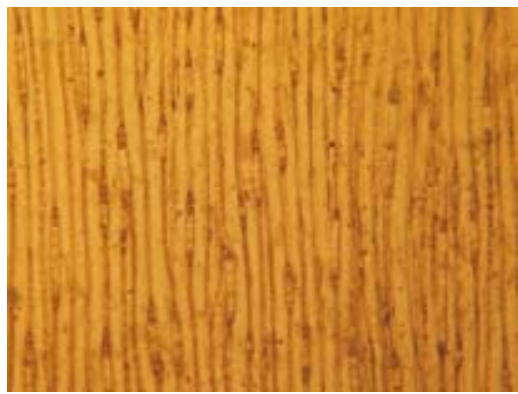

Plano Tangencial $<13.1 \mathrm{x}>$

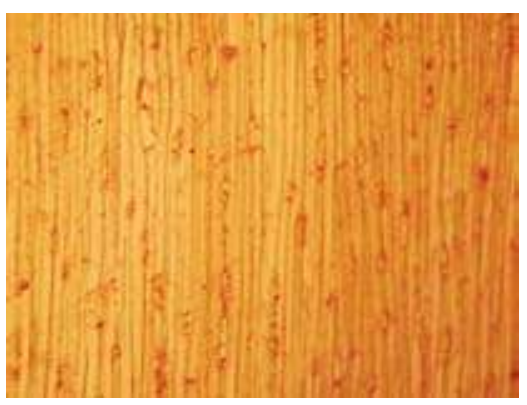

Plano Tangencial $<10.1 \mathrm{x}>$

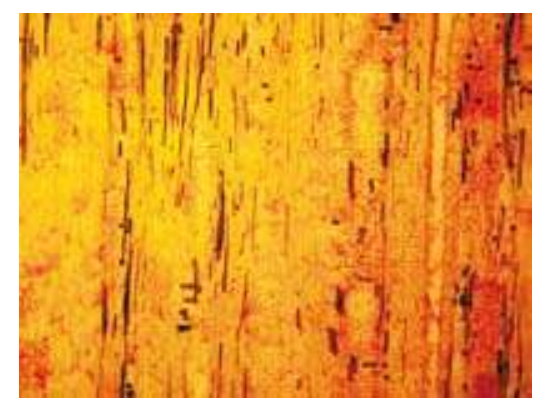

Plano Radial $<9 x>$

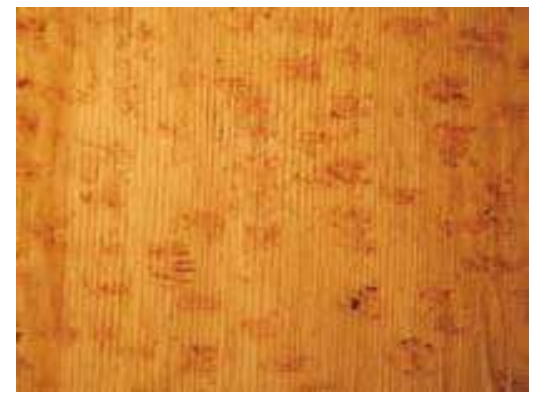

Plano Radial $<9 \mathrm{x}>$

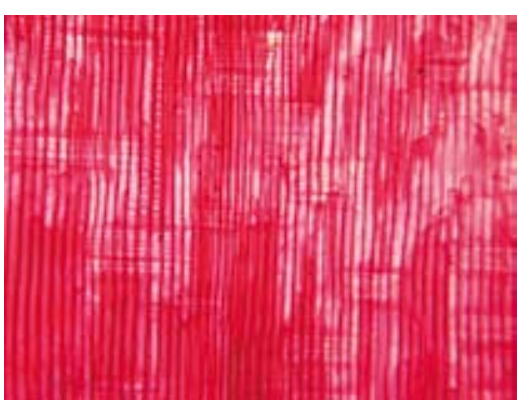

Plano Radial $<10.1 \mathrm{x}>$ 
